\title{
Characterizing Optical Filters for Wildfire Detectors
}

\author{
N. P Mashaba and D. J Griffith
}

\begin{abstract}
Near infrared detectors have gained popularity, mainly in the military, because of their capability of detecting wildfires. The Council for Scientific and Industrial Research (CSIR) focuses on the development and deployment of wildfire detectors within the Southern hemisphere, specifically in Pretoria. These detectors employ ultra narrow bandpass filters which highly transmit desired wavelengths including near-infrared regimes, where wildfire detection is possible. However, these filters are dependant on environmental conditions such as temperature change. Temperature variations cause sensitivity on the angle of incidence and wavelength shift, which reduces filter transmission. Here, we characterize optical filters which can be integrated to the CSIR's wildfire detectors. We select the best performing optical filters which optimally transmit and $770 \mathrm{~nm}$ and block unwanted wavelengths at different angles of incidence and temperatures.
\end{abstract}

\section{Keywords - Bandpass filters, near infrared imaging.}

\section{INTRODUCTION}

A near infrared (NIR) camera (Kline) is under development at the CSIR. This camera will be used to detect wildfires in the Southern hemisphere. NIR detectors have become the most commonly used traditional devices to detect and monitor wildfires [1-7]. NIR ranges from 700 to $1400 \mathrm{~nm}$ and is very sensitive to reflected sunlight and emitted energy sources. The ability to detect wildfires highly depends on the optical filters used.

The main filters which are used in the NIR camera are the ultra narrow bandpass filters. An ultra narrow bandpass filter has the ability to transmit frequencies within a certain range and reject frequencies outside that range (see Fig. 1). As light makes its way through the optical filter, its direction changes as it passes from one layer to the next, resulting in internal interference [8], [9]. This is due to the differences between the refractive indices of the materials in the dielectric thin-film coating. The configuration of the layers results in an optical filter that manipulates different wavelengths of light in different ways. Depending on the wavelength and type of optical filter, light can be reflected off of the filter, transmitted through it, or absorbed by it. Although it is generally desirable

Manuscript received 10 June, 2020

This work was supported by the Optronics Sensor Systems (OSS) group at the CSIR.

N. P Mashaba is with Optronics Sensor Systems department at The Council for Scientific and Industrial Research (CSIR), Defence and Security Cluster, P. O. Box 395, Pretoria, South Africa,

D. J Griffith is with Hensoldt Optronics, Nellmapius Dr, Highveld, Centurion, 0157. to have normal incidence at the filter (i.e., collimated light where an optical filter is used), other system design (such as, lenses) considerations may take precedence. Parameters such as angle of incidence, operating temperature, illumination source, and detector are vulnerable upon filter performance. The wavelength shift in a filter is impacted by the refractive index of "air", which is not always clearly defined and cannot be predicted.

In this paper, we characterize ultra narrow bandpass filters. We explore the key method used to determine the shift factors for each angle of incidence making up a ray bundle, using filters. The spectral shift with respect to the angle exhibited by the ultra-narrow band spectral filter will likely be a potent constraint on FOV. Hence, this effect is analysed in considerable detail. Moreover, we will select two most outperforming interference filters, where each will be used to transmit and block (for probing) $770 \mathrm{~nm}$ wavelength for the Kline detectors.

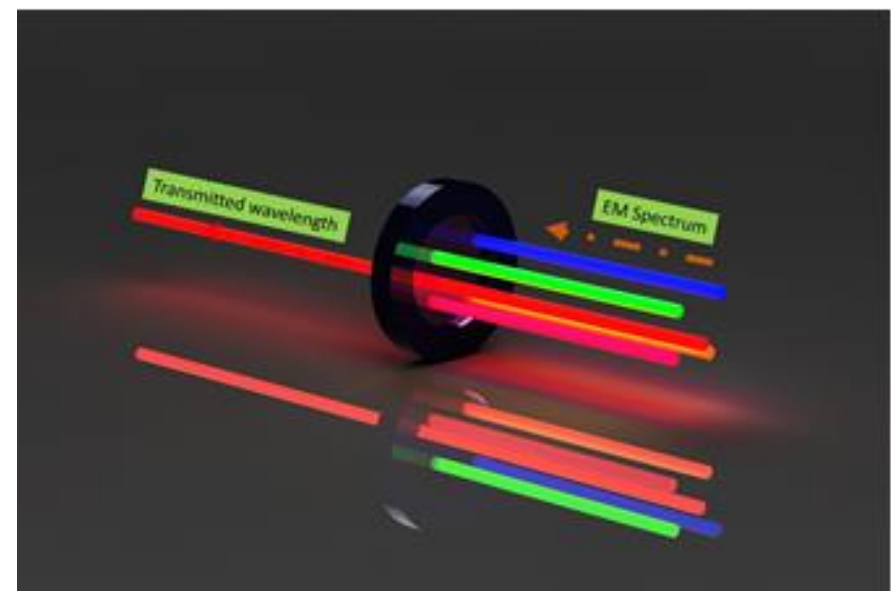

Fig. 1. A demonstration of an ultra narrow bandpass filter blocking a range of wavelengths while transmitting a single wavelength (e.g. 770 $\mathrm{nm})$.

\section{TEMPERATURE ShifT SimUlation}

The refractive indices of glass and other optical materials also change with temperature. This introduces risks that the imaging channels can also go out of focus with any change in temperature as well as any change in ambient pressure. How can we select a resilient filter which highly transmit or block light while the temperature is varied? Interestingly, one can compute a wavelength shift of each filter to check the cut-off frequency using [10], [11]

$$
\lambda\left({ }^{\circ} C\right)=\left(\lambda_{0}\right)+\Delta T\left(\Delta \lambda_{0} \mu\left({ }^{\circ} C\right)\right)
$$

where $\lambda 0$ is the initial wavelength, $\Delta \mathrm{T}$ is the change in 
temperature and $\Delta \lambda 0$ is the wavelength coefficient.

Figure 2 shows the spectral shift that we simulated using data obtained from Alluxa. Here, the wavelength coefficient we used is

$0.0049 \mathrm{~nm}$ and is recommended by Alluxa for every specific filter used here. The filters, $769.89 \mathrm{~nm}$ and $770.0 \mathrm{~nm}$ were analysed for the transmision of the Kline whereas, $757.9 \mathrm{~nm}$ and $732 \mathrm{~nm}$ are for blocking the Kline emission. Here we varied the temperatures from

-80 to $40 \circ$ in a steps of $20^{\circ}$. It is see that all the filters vary the same way because of the similarity of the coefficient. However, it is seen that at the transmission values are dependant on the transmission band of the filter.

Geometrical optics describes light propagation in terms of rays.
The ray in geometric optics is useful in approximating the path along which light propagates in space. The simplification of geometrical optics include light rays propagating in rectilinear paths as they travel in a homogeneous medium, follow curved paths in a medium in which the refractive index changes and may be absorbed or reflected. Fig. 3 shows a transmission of the geometrical rays (chief and marginal ray), simulated using all bandpass filters and a lens with different $f$ -number values ( $f=1.8$ and 5.5). In parallel, we show the average mean which result from the geometrical rays. It is seen (in Fig.3 (a)-(d)) that a smaller f-number cuts off the higher order frequency, this means that there will only be less transmission of all the light. Fig. 3 (e) and (f), show that there is zero transmission in the $770 \mathrm{~nm}$ wavelength.
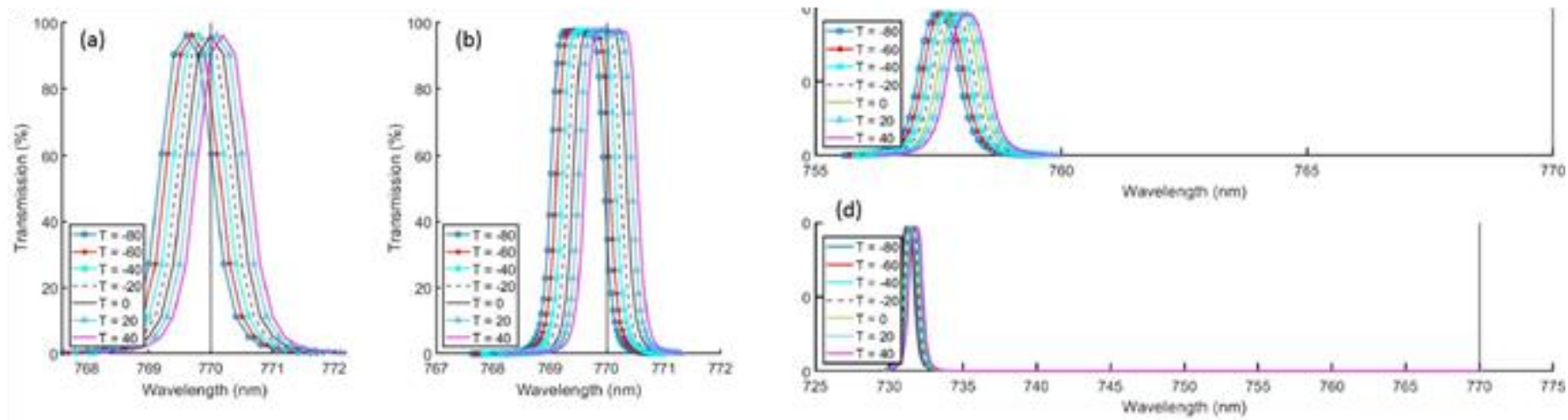

Fig. 2. Spectral shifts for the Alluxa narrow band filters with $0.0049 \mathrm{~nm}$ coefficient. (a) Transmission of a 770-10 OD4 bandpass filter, showing different transmission levels. (b) A 769.89-1 OD7 ultra narrow bandpass filter at different temperatures (having a wide bandwidth and a flat top). The symbol T, refers to the change in temperature and is measured in degrees. The black solid line represents the Kline emission wavelength $(770 \mathrm{~nm})$. (c) Transmission of a

757.9-1 OD4 bandpass filter, showing different transmission levels. (d) A 732.0-1 OD4 ultra narrow bandpass filter at different temperatures. The symbol $\mathrm{T}$, refers to the change in temperature and is measured in degrees.
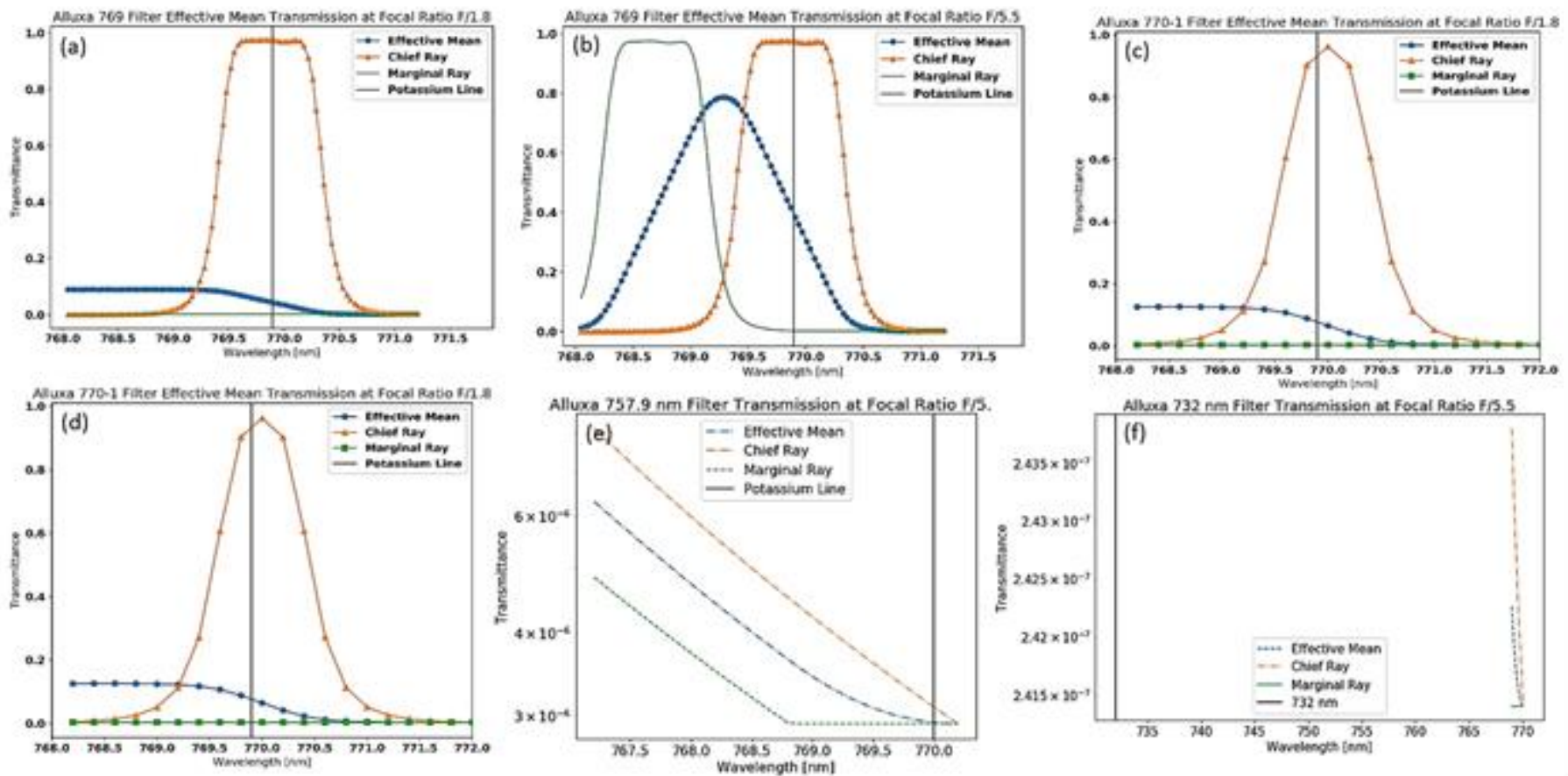

Fig. 3. Transmission of geometrical ray for (a)-(b) Alluxa $769 \mathrm{~nm}$, (c)-(d) $770 \mathrm{~nm}$, (c) $757.9 \mathrm{~nm}$, and (f) $732 \mathrm{~nm}$ bandpass filter with f /\# = 1.8 , $\mathrm{f}$ /\# = 5.5, for $769 \mathrm{~nm}$ and $770 \mathrm{~nm}$, and only $\mathrm{f} / \#=5.5$ for $757.89 \mathrm{~nm}$ and $732 \mathrm{~nm}$ 
Now that we have calculated the wavelength shift, we can simulate the transmission of each filter. When placed in a convergent light bundle of a particular cone half-angle (CHA, symbol $\varphi c$ ), there is both a shift in effective centre wavelength as well as a change in the shape of the bandpass function. The resulting transmittance function of the bundle of rays is given by

$$
T(\lambda, \varphi)=T\left(\lambda+\Delta \lambda_{\varphi}\right)
$$

where $\varphi$ is the angle of incidence and $\Delta \lambda \theta$ is the wavelength change due to angles of incidence computed from [12], [13]

$$
\Delta \lambda_{\phi}=\lambda_{0} \sqrt{1-\left(\frac{\sin ^{2} \phi}{n_{e f f}^{2}}\right)} .
$$

Here $\mathrm{n}_{\text {ef }}$ is the effective refractive index of the optical filter.

The numerical aperture of the convergent cone, $\alpha=\sin \varphi$ corresponds to a projected solid angle of $\Omega=\pi \sin ^{2} \varphi=\pi \alpha^{2}$. The differential increase in solid angle is $\mathrm{d} \Omega=2 \pi \alpha \mathrm{d} \alpha$. The effective transmittance function $\tau(\lambda, \varphi)$ for the whole cone of CHA $\varphi c$ is an integral of the transmittance function for normal incidence $\tau(\lambda)$ over the CHA, wavelength-shifted as above, weighted by the differential solid angle. That is

$$
\tau(\lambda ; \phi) \propto \int_{0}^{\phi_{c}} \tau(\lambda, \alpha) d \Omega .
$$

This leads to

$$
\tau(\lambda ; \phi) \propto \int_{0}^{\alpha_{c}} \tau(\lambda, \alpha) 2 \pi \alpha d \alpha
$$

where $\alpha_{c}=\sin \varphi_{c}$. The required normalisation is the integrated weighting of the transmittance function, which is

$$
\int_{0}^{\alpha_{c}} 2 \pi \alpha d \alpha=\pi \alpha_{c}^{2}
$$

Giving

$$
\tau\left(\lambda, \phi_{c}\right)=\frac{2}{\alpha_{c}^{2}} \int_{0}^{\alpha_{c}} \tau(\lambda, \alpha) \alpha d \alpha .
$$

Fig. 4 show the transmission of light at different angles of incidence and temperatures. The transmission is much higher at Fig. 4(a) and (b). However, transmission drops (at about 4 degrees) much earlier with $769 \mathrm{~nm}$ filter, whereas with $770 \mathrm{~nm}$, the transmission drop is seen at about 5 degrees angle of incidence. Note that there is also a zero transmission with 757 $\mathrm{nm}$ and $732 \mathrm{~nm}$. We can see that $732 \mathrm{~nm}$ filter transmits much less light than $757 \mathrm{~nm}$ filter.
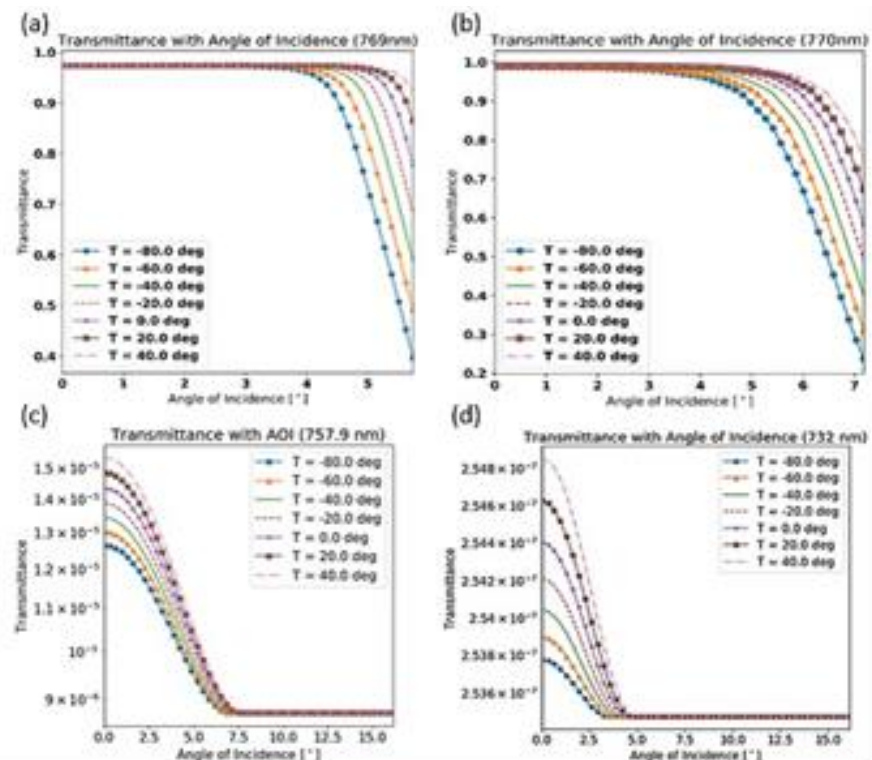

(d)

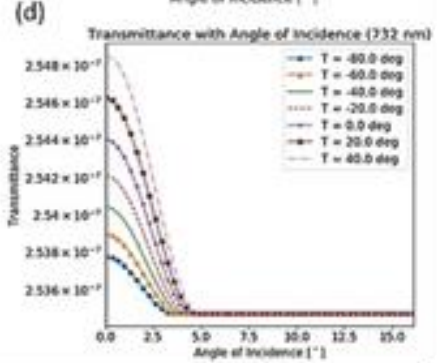

Fig. 4. Transmission of light at different angles of incidence and temperatures with (a) $769 \mathrm{~nm}$, (b) $770 \mathrm{~nm}$, (c) $757.9 \mathrm{~nm}$, and (d) 732 nm ultra narrow band pass filter.

\section{CONCLUSIONS}

Here, we characterized bandpass filters by varying the temperature and we simulated the transmission at difference angles of incidence. We compared the wavelength shift of all the bandpass filters. For transmitting potassium, we used $769.89 \mathrm{~nm}$ and $770.1 \mathrm{~nm}$ bandpass filter. And for blocking the kline emission, we used $757.9 \mathrm{~nm}$ and $732 \mathrm{~nm}$. As a result we found that, the filters have peak transmissions of 0.97 and 0.99 at $770 \mathrm{~nm}$. Moreover they are both centered at wavelengths of $770 \mathrm{~nm}$ with bandwidths of $1 \mathrm{~nm}$. Both filters have the same temperature coefficients and therefore should respond the same to higher and lower temperatures. The disparity in their spectral profiles contributes to the observed differences in the transmission. As such, the $770 \mathrm{~nm}$ out performs the $769 \mathrm{~nm}$ filters due to the higher transmission. The $769 \mathrm{~nm}$ filter, however, shows a constant transmittance for AOI of up to 5 degrees at various temperatures while the $770 \mathrm{~nm}$ filter shows a slow decay but still maintaining a high transmission. As such the $770 \mathrm{~nm}$ filter shows a better performance. We therefore, recommend the $770 \mathrm{~nm}$ filter because of its higher transmission. Moreover, we found that the filters to block Kline have peak transmissions of about $10-5$ and $10-7$ at $770 \mathrm{~nm}$. The $732 \mathrm{~nm}$ out performs the $757.9 \mathrm{~nm}$ filters due to the lower transmission. As such, we recommend the $732 \mathrm{~nm}$ filter for probing $770 \mathrm{~nm}$ wavelength because of its lower transmission.

We further compared lenses of different $\mathrm{f}$-number values to calculate the effective transmission of light. We observed a cut off frequency for a lens with $\mathrm{f} / 1.8$ at $770 \mathrm{~nm}$. And for a lens with index $\mathrm{f} / 5.5$, the transmission of the geometrical rays is above $90 \%$. As such, we recommend a lens which has an index of $f / 5.5$ because of their high transmission of rays which will certainly reduce aberrations on the system. 


\section{REFERENCES}

[1] Y. Le Maoult, T. Sentenac, J.-J. Orteu, and J.-P. Arcens, "Fire detection: a new approach based on a low cost ccd camera in the near infrared," Process Safety and Environmental Protection, vol. 85, no. 3, pp. 193-206,

2007.

https://doi.org/10.1205/psep06035

[2] E. Magidimisha and D. J. Griffith, "Remote optical observations of actively burning biomass fires using potassium line spectral emission," in Fourth Conference on Sensors, MEMS, and Electro-Optic Systems, vol. 10036, p. 1003611, International Society for Optics and Photonics, 2017.

[3] J. Everitt, D. Escobar, and M. Davis, "Mid-infrared video: A possible tool for thermal analysis of wildfires," Geocarto International, vol. 4, no. 4, pp. 39-45, 1989. https://doi.org/10.1080/10106048909354233

[4] P. H. Greenfield, W. Smith, and D. C. Chamberlain, "Phoenix-the new forest service airborne infrared fire detection and mapping system," in 2nd Int. Wildland Fire Ecology and Fire Management Congress and the 5th Symposium on Fire and Forest Meteorology, 2003.

[5] R. S. Allison, J. M. Johnston, G. Craig, and S. Jennings, "Airborne optical and thermal remote sensing for wildfire detection and monitoring," Sensors, vol. 16, no. 8, p. 1310, 2016. https://doi.org/10.3390/s16081310

[6] J. D. Burnett and M. G. Wing, "A low-cost near-infrared digital camera for fire detection and monitoring," International Journal of Remote Sensing, vol. 39, no. 3, pp. 741-753, 2018. https://doi.org/10.1080/01431161.2017.1385109

[7] E. Magidimisha and D. J. Griffith, "Measurement of the thermal-vacuum defocus of an objective lens for an imaging payload on a cubesat," in Fifth Conference on Sensors, MEMS, and Electro-Optic Systems, vol. 11043, p. 1104318, International Society for Optics and Photonics, 2019. https://doi.org/10.1117/12.2502328

[8] O. E. DeLange, "Wide-band optical communication systems: Part ii-frequency-division multiplexing," Proceedings of the IEEE, vol. 58, no. 10, pp. 1683-1690, 1970. https://doi.org/10.1109/PROC.1970.7989

[9] P. Grangier, R. Slusher, B. Yurke, and A. LaPorta, "Squeezed-light- enhanced polarization interferometer," Physical Review Letters, vol. 59, no. 19, p. 2153, 1987. https://doi.org/10.1103/PhysRevLett.59.2153

[10] Newport, "Specifying bandpass filters," 2020.

[11] T. Stolberg-Rohr and G. J. Hawkins, "Spectral design of temperature- invariant narrow bandpass filters for the mid-infrared," Optics Express, vol. 23, no. 1, pp. 580-596, 2015. https://doi.org/10.1364/OE.23.000580

[12] O. thin-films Alluxa team, "Angle of incidence and polarization," 2020. [13] J. L. Rienstra, "Transformation of filter transmission data for f-number and chief ray angle," in Infrared Imaging Systems: Design, Analysis, Modeling, and Testing IX, vol. 3377, pp. 267-275, International Society for Optics and Photonics, 1998 